\title{
Threshold Policy for Global Games with Noisy Information Sharing
}

\author{
Hessam Mahdavifar, Ahmad Beirami, Behrouz Touri, and Jeff S. Shamma
}

\begin{abstract}
It is known that global games with noisy sharing of information do not admit a certain type of threshold policies [1]. Motivated by this result, we investigate the existence of threshold-type policies on global games with noisy sharing of information and show that such equilibrium strategies exist and are unique if the sharing of information happens over a sufficiently noisy environment. To show this result, we establish that if a threshold function is an equilibrium strategy, then it will be a solution to a fixed point equation. Then, we show that for a sufficiently noisy environment, the functional fixed point equation leads to a contraction mapping, and hence, its iterations converge to a unique continuous threshold policy.
\end{abstract}

\section{INTRODUCTION}

Games of incomplete information are central to modeling of socio-economical behaviors in social networks. In games with incomplete information, the information that is shared among the agents is not symmetric and often each agent has access to limited information about the game's parameters. This incompleteness of information is often represented as functionality of the payoff function on some random variable whose exact value is not known to the agents.

A subclass of games with incomplete information is the class of global games where, in its simplest form, each agent takes either of the two actions: safe or risky action. The payoff of an agent taking the risky action depends monotonically increasingly on how many other agents take the risky action and is a decreasing function of an underlying economical or political fundamental. Global games were originally introduced in [2], [3] and since then have been used to model and study many models of social coordination phenomena including bank runs, currency crisis, and political uprisings (see [4] and the references therein).

The majority of the past studies on global games have been focused on the information structure where each agent has an independent noisy observation of the underlying economical or social fundamental, i.e., given the economical fundamental, the observations of each agent is independent of the rest of the agents' observations. In such settings, it has been shown that, under some condition on the distribution of the underlying fundamental and the independency of agents' observations, there exists an equilibrium strategy

The work of J. S. Shamma was supported by AFOSR/MURI Projects No. FA9550-09-1-0538 and No. FA9550-10-1-0573.

H. Mahdavifar is with the Department of Electrical and Computer Engineering, University of California San Diego, USA (email: hessam@ucsd.edu)

A. Beirami is with the Department of Electrical and Computer Engineering, Duke University, USA, and also with the Research Laboratory of Electronics, Massachusetts Institute of Technology, USA (email: ahmad.beirami@duke.edu, beirami@mit.edu).

B. Touri is with the Department of Electrical, Computer, and Energy Engineering, University of Colorado Boulder, USA (email: behrouz.touri@colorado.edu).

J. S. Shamma is with the School of Electrical and Computer Engineering, Georgia Institute of Technology, USA, and also with the CEMSE Division, King Abdullah University of Science and Technology, Saudi Arabia (email: shamma@gatech.edu,jeff.shamma@kaust.edu.sa). with threshold policies based on the private observations of each agent. In [5], the case of perfect sharing of information was introduced and the structure of equilirbia for global games with perfect sharing of information was discussed. In [1], global games with noisy sharing of information was introduced where two agents share their information over a noisy channel with one another and, there, it is shown that a certain intuitive threshold policy with noisy sharing of information does not exist.

In this work we show the existence of threshold policies in a broader sense for global games with noisy sharing of information when the noise parameters and the information structure of the model satisfies a given condition. To show this result, we first relate the existence of a threshold strategy (within the given class of threshold policies) to the existence of a solution to a functional equation. We then utilize Banach fixed point theorem and show that such an equilibrium exists if the noise and the information structure of the underlying model satisfy the given conditions. This work also relates to many of the current attempts for understanding the role of information and information structure in multiagent decision making problems [6]-[9].

The structure of this paper is as follows: in Section II we formally introduce global games with noisy sharing of information, and we mathematically formulate the problem of interest. In Section III we discuss the main existence theorem and its proof. Furthermore, we analyze the iterative scheme to find the threshold policy. Finally, we conclude the paper and provide few directions for further studies in Section IV.

\section{Problem Setup}

In this section, we present the framework of the problem that will be studied in this paper. We study the basic form of the global games with noisy sharing of information. In this setting, we have a set $[n]:=\{1, \ldots, n\}$ of $n$ agents or players and each agent has a set of binary actions $A_{i}=\{0,1\}$. We refer to the action $\alpha_{i}=1$ as the risky action and $\alpha_{i}=0$ as the safe action. The payoff of an agent taking the safe action is zero whereas the payoff of an agent taking the risky action is $\sum_{i=1}^{n} \alpha_{i}-\theta$ where $\theta$ is a random variable representing the underlying fundamental in the society. In other words, if $\alpha=\left(\alpha_{1}, \ldots, \alpha_{n}\right) \in\{0,1\}^{n}$ is an action profile of the $n$ agents, then the utility of the $i$ th agent is the function $u_{i}$ with

$$
u_{i}\left(\alpha_{i}\right)=\alpha_{i}\left(\sum_{j=1}^{n} \alpha_{j}-\theta\right) .
$$

If all the agents know what the exact value of $\theta$ is, the game would be a game with perfect information and if $\theta \neq$ $n$, either of the coordinated actions $(0, \ldots, 0)$ or $(1, \ldots, 1)$ would be appealing. The major challenge in the study of such a coordination game is that the fundamental $\theta$ is not known exactly to any of the players and each of the players have a noisy perception of $\theta$. One can think of such a game as a 


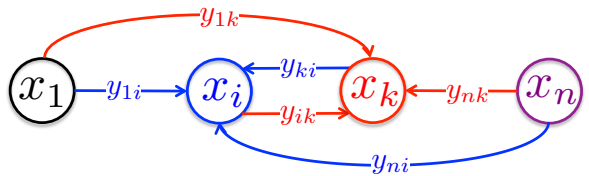

Figure 1. Communication flow.

model for political uprisings where $\theta$ represents the strength of the political regime. Hence, $\sum_{i=1}^{n} \alpha_{i}$ is the number of people that take the risky action against the regime.

Observations and Policies: In the above model, none of the agents is observing $\theta$ directly and each of them observes a noisy observation of $\theta$ through which they have to make a decision. Mathematically, we represent agent $i$ 's observation by a random vector $y_{i} \in \mathbb{R}^{d_{i}}$ (which relates to $\theta$ and other agents' observations). We refer to $y_{i}$ as the (private) information of agent $i$ (about $\theta$ ). We refer to a measurable function $s_{i}: \mathbb{R}^{d_{i}} \rightarrow A_{i}$ that maps a private observation of agent $i$ to one of the two actions as a (pure) strategy or policy. When $d_{i}=1$ for some $i$, we say that $s_{i}$ is a threshold policy if $s_{i}(y)=1$ for $y \leqslant t$ and $s_{i}(y)=0$ for $y>t$ for some threshold value $t$. We denote such a strategy by $s_{i}(y)=1_{y \leqslant t}$. In almost all the instances of the global games, the random variables have continuous joint distribution and and hence, the value of the strategy $s_{i}$ at the threshold value $t$ is practically unimportant.

Equilibrium: Our focus in this paper is on the existence of a strategy profile $s=\left(s_{1}, \ldots, s_{n}\right)$ that results in a Bayesian Nash Equilibrium. To introduce this, let $s=\left(s_{1}, \ldots, s_{n}\right)$ be a strategy profile of the $n$ agents and let $s^{-i}=\left(s_{1}, \ldots, s_{i-1}, s_{i+1}, \ldots, s_{n}\right)$ be the strategy profile of the $n-1$ agents except the $i$ th agent's strategy. We say that a best response strategy to the strategy profile $s^{-i}$ is a strategy $\tilde{s}: \mathbb{R}^{d_{i}} \rightarrow A_{i}$ such that $\tilde{s}(u)=0$ if

$$
1+E\left[\sum_{j \neq i} s_{j}\left(y_{j}\right) \mid y_{i}=u\right]<E\left[\theta \mid y_{i}=u\right]
$$

and $\tilde{s}(u)=1$ if

$$
1+E\left[\sum_{j \neq i} s_{j}\left(y_{j}\right) \mid v_{i}=u\right]>E\left[\theta \mid y_{i}=u\right] .
$$

In other words, the best response strategy for the $i$ th agent is to take action 1 if the expected value of its payoff, given its observation $y_{i}$, is positive and to take action 0 otherwise. We denote the set of all best responses to a strategy profile $s^{-i}$ by $B R_{i}\left(s^{-i}\right)$. Finally, we say that a strategy profile $s=$ $\left(s_{1}, \ldots, s_{n}\right)$ is a Bayesian Nash Equilibrium or simply an equilibrium if $s_{i} \in B R\left(s^{-i}\right)$ for all $i \in[n]$.

An extensively studied model in global games is the case where $d_{i}=1$ for all $i \in[n]$ and $y_{i}=x_{i}=\theta+\xi_{i}$ where $\left\{\xi_{1}, \ldots, \xi_{n}\right\}$ are independent and identically distributed $\mathcal{N}\left(0, \sigma^{2}\right)$ Gaussian random variables for some $\sigma^{2}>0$. Furthermore, it is customary to assume that $\theta$ is picked from a non-informative uniform distribution over $\mathbb{R}$. See [4] and the references therein for further discussion on this assumption.

In [4], it is shown that there exists a symmetric threshold policy on $x_{i}$ s which corresponds to a Bayesian Nash equilibrium for this instance of global games. In other words, there exists a threshold value $t \in \mathbb{R}$ such that for $x_{i} \leqslant t$, agent $i$ chooses to take the risky action $\left(a_{i}=1\right)$ and for $x_{i}>t$,

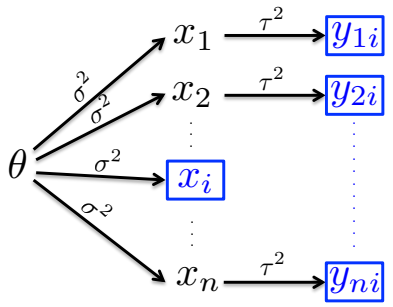

Figure 2. The dependence diagram between the observation vector $y_{i}=$ $\left(x_{i},\left\{y_{j i}\right\}_{j \in[n] \backslash\{i\}}\right)$ of agent $i$. In this diagram, $x \stackrel{\sigma^{2}}{\longrightarrow} y$ implies that $y=x+z$, where $x$ and $z$ are independent and $z \sim \mathcal{N}\left(0, \sigma^{2}\right)$.

she takes the safe action and such an action profile leads to an equilibrium. Note that $x_{i}=E\left[\theta \mid x_{i}\right]$ which means that in such an equilibrium each agent should compare her expected strength of regime given her private observation to a threshold and take a proper action accordingly.

In [1], a simple instance of global games with $n=2$ agents was studied where two agents perform information sharing and it is shown that the above intuitive equilibrium does not exist in this case, i.e., there doesn't exist a threshold policy equilibrium based on the expected strength of the regime given each agent's observations.

Our Model: In this work, we consider a more general case of the global games considered in [1] where $n$ agents have $n$ independent observations $\left(x_{1}, \ldots, x_{n}\right)$ of $\theta$ and they share their private observation with other agents through a noisy channel. More precisely, throughout this work the focus will be on global games with $n$ agents where the utility of each agent is given by (1) and the information structure satisfies the following assumption.

Assumption 1 We assume that $\theta$ is uniformly distributed over $\mathbb{R}$. Also, agent $i$ 's private information is the $n$ dimensional random vector:

$$
y_{i}=\left(x_{i},\left\{y_{j i}\right\}_{j \in[n] \backslash\{i\}}\right),
$$

where $x_{i}=\theta+\xi_{i}$ for all $i \in[m]$ and $y_{j i}=x_{j}+\zeta_{j i}$ for $j \neq i$, where $\xi_{1}, \ldots, \xi_{n}$ are i.i.d. $\mathcal{N}\left(0, \sigma^{2}\right)$ random variables and $\zeta_{j i}$ are i.i.d. $\mathcal{N}\left(0, \tau^{2}\right)$ random variables that are independent of $\xi_{1}, \ldots, \xi_{n}$ and $\sigma^{2}, \tau^{2}>0$ are given parameters.

The information communication flow is depicted in Figure 1. Note that the information of the agents about $\theta$ through noisy observations is no longer independent which creates further complication. The dependence diagram of the random observation vector $y_{i}=\left(x_{i},\left\{y_{j i}\right\}_{j \in[n] \backslash\{i\}}\right)$ that is available to agent $i$ is shown in Figure 2.

Throughout the rest of the paper, the scalar $\sum_{j \in[n] \backslash\{i\}} y_{j i}$ which is the sum of all the information arrived at agent $i$ from the rest of agents plays a central role. We denote this quantity by $z_{i}$, i.e.,

$$
z_{i}:=\sum_{j \in[n] \backslash\{i\}} y_{j i} .
$$

We define a global game with noisy sharing of information as bellow.

Definition 1 We refer to a game with utility functions $u_{1}, \ldots, u_{n}$ described by (1) and information structure satisfying Assumption 1 as a global game with noisy information sharing. 
The following results present the generalization of Lemma 1 of [1] to the above multiple agent setting. The first lemma derives the probability distribution of $\theta$ conditioned on the observation vector $y_{i}$.

Lemma 1. Suppose that Assumption 1 holds. Let $\eta_{n}^{2}$ be a scalar such that

$$
\frac{1}{\eta_{n}^{2}}=\frac{1}{\sigma^{2}}+\frac{n-1}{\sigma^{2}+\tau^{2}}
$$

Further, let $a_{n}:=\frac{\eta_{n}^{2}}{\sigma^{2}}$ and $b_{n}:=\frac{\eta_{n}^{2}}{\sigma^{2}+\tau^{2}}$. Then, conditioned on agent is observation $y_{i}, \theta$ is given by

$$
\theta=a_{n} x_{i}+b_{n} z_{i}+\varepsilon_{\theta},
$$

where $\varepsilon_{\theta}$ is a $\mathcal{N}\left(0, \eta_{n}^{2}\right)$ Gaussian random variable independent of $y_{i}$.

Proof. Note that $\left(x_{i},\left\{y_{j i}\right\}_{j \in[n] \backslash\{i\}}\right)$ are zero-mean independent Gaussian observations of $\theta$. Therefore, the result follows by invoking Lemma 8 from the appendix.

To investigate the existence of a threshold policy for agent $i$, we further need to derive the distribution of agent $k$ 's observation vector $y_{k}$ given agent $i$ 's observation $y_{i}$. In a sense, this is agent $i$ 's perception of what is available to agent $k$.

Lemma 2. Let Assumption 1 hold. Further, let

$$
\frac{1}{\gamma_{n}^{2}}=\frac{1}{\tau^{2}}+\frac{1}{\eta_{n-1}^{2}+\sigma^{2}}
$$

and define $c_{n}:=\frac{\gamma_{n}^{2}}{\eta_{n-1}^{2}+\sigma^{2}}$ and $d_{n}:=\frac{\gamma_{n}^{2}}{\tau^{2}}$. Then, conditioned on $y_{i}$, we can write $y_{k}=\left(x_{k},\left\{y_{l k}\right\}_{l \in[n] \backslash\{k\}}\right)$ as jointly Gaussian random variables defined by:

$$
x_{k}=\overline{x_{k}}+\varepsilon_{k}, \quad y_{i k}=x_{i}+\varepsilon_{i k}, \quad y_{l k}=\overline{y_{l k}}+\varepsilon_{l k},
$$

where $^{1}$

$$
\left\{\begin{array}{l}
\overline{x_{k}}=c_{n} a_{n-1} x_{i}+d_{n} y_{k i}+c_{n} b_{n-1} \sum_{j \in[n] \backslash\{i, k\}} y_{j i}, \\
\overline{y_{l k}}=c_{n} a_{n-1} x_{i}+d_{n} y_{l i}+c_{n} b_{n-1} \sum_{j \in[n] \backslash\{i, l\}} y_{j i},
\end{array}\right.
$$

and $\left(\varepsilon_{k},\left\{\varepsilon_{l k}\right\}_{l \in[n] \backslash\{k\}}\right)$ are jointly Gaussian random variables independent of $\left(x_{i},\left\{y_{j i}\right\}_{j \in[n] \backslash\{i\}}\right)$, and $\varepsilon_{k}$ is $\mathcal{N}\left(0, \gamma_{n}^{2}\right)$; and $\varepsilon_{i k}$ is $\mathcal{N}\left(0, \tau^{2}\right)$ independent of $\epsilon:=\left(\varepsilon_{k},\left\{\varepsilon_{l k}\right\}_{l \in[n] \backslash\{i, k\}}\right)$.

Proof. Since $\theta$ follows a non-informative uniform prior on $\mathbb{R}, x_{k}$ (or $y_{j k}$ ) also follows a uniform prior on $\mathbb{R}$. Therefore, $\left(x_{i},\left\{y_{j i}\right\}_{j \in[n] \backslash\{i\}}\right)$ can be viewed as zero-mean independent Gaussian observations of $x_{k}$ (or $y_{j k}$ ). Hence, by invoking Lemma 8 we arrive at the desired results. Furthermore, it is clear that, conditioned on $x_{i}, y_{i k}$ is independent of all other $\left\{x_{j}\right\}_{j \in[n] \backslash\{i\}}$ and $\left\{y_{j k}\right\}_{j \in[n] \backslash\{i\}}$. Hence, $\varepsilon_{i k}$ is independent of $\left(\varepsilon_{k},\left\{\varepsilon_{l k}\right\}_{l \in[n] \backslash\{i, k\}}\right)$ as desired.

\section{MAin RESUlT}

In this section, we discuss a result on the existence of an equilibrium within a class of equilibrium policies for the global games introduced in the previous section. This equilibrium can be described as a threshold policy and we regard it as a fixed point of a certain operation. We further provide a constructive framework for convergence to this fixed point solution.

\footnotetext{
${ }^{1}$ Note that $\overline{x_{k}}$ and $\overline{y_{l k}}$ are functions of the observation vector $y_{i}$ of agent $i$ but the dependence is left implicit for brevity.
}

\section{A. A class of threshold policies: definition and existence}

We start by introducing the set of threshold policies that we will be focusing on. Consider the information available to agent $i$, i.e. $y_{i}=\left(x_{i},\left\{y_{j i}\right\}_{j \in[n] \backslash\{i\}}\right)$. For a function $h$ : $\mathbb{R} \rightarrow \mathbb{R}$, we will be focusing on the coordinated threshold strategies $1_{z_{i} \leqslant h\left(x_{i}\right)}$ where $z_{i}$ is given by (2). The goal is to show there exists, under some conditions on noise parameters $\sigma^{2}, \tau^{2}$, a decreasing function $h: \mathbb{R} \rightarrow \mathbb{R}$, such that the strategy profile $s=\left(1_{z_{1} \leqslant h\left(x_{1}\right)}, \ldots, 1_{z_{n} \leqslant h\left(x_{n}\right)}\right)$ is an equilibrium for the global games with noisy sharing of information. In other words, a threshold policy $s_{i}=1_{z_{i} \leqslant h\left(x_{i}\right)}$ is the policy that prescribes:

$$
\begin{cases}\alpha_{i}=1 & \text { if } z_{i} \leqslant h\left(x_{i}\right) \\ \alpha_{i}=0 & \text { if } z_{i}>h\left(x_{i}\right)\end{cases}
$$

for agent $i$. We refer to such a function $h$ as a threshold function and we refer to the resulting coordinated strategy profile

$$
s=\left(1_{z_{1} \leqslant h\left(x_{1}\right)}, \ldots, 1_{z_{n} \leqslant h\left(x_{n}\right)}\right)
$$

as a coordinated threshold policy. If (5), is an equilibrium for the underlying game, we say that the threshold function $h$ leads to a (coordinated) threshold policy equilibrium.

By the definition of a (Bayesian Nash) equilibrium, the function $h$ leads to a threshold policy equilibrium if a best response of any agent $i \in[n]$ is characterized by the threshold policy described in (4). In other words, we have

$$
1+\sum_{k \in[n] \backslash\{i\}} P\left(z_{k} \leqslant h\left(x_{k}\right) \mid y_{i}\right) \geqslant E\left[\theta \mid y_{i}\right]=a_{n} x_{i}+b_{n} z_{i}
$$

if and only if $z_{i} \leqslant h\left(x_{i}\right)$, for any $i \in[n]$.

Remark. It is shown in [1] that an equilibrium with a threshold policy on $E\left[\theta \mid y_{i}\right]$ does not exist for $n=2$. In light of the definition of threshold functions provided here, this results can be stated as there is no threshold function $h(x)=$ $-\frac{a_{n}}{b_{n}} x+c$, where $c \in \mathbb{R}$ is a constant. We emphasize that our definition of a threshold function only considers a coordinated threshold policy (if it exists), where all agents take actions according to the same threshold function $h$.

Assuming that the threshold function $h$ is continuous, one can observe that if $h\left(x_{i}\right)=z_{i}$, then (6) also turns into equality. In other words, we have

$$
a_{n} x_{i}+b_{n} h\left(x_{i}\right)=1+\sum_{k \in[n] \backslash\{i\}} P\left(z_{k} \leqslant h\left(x_{k}\right) \mid y_{i}\right) .
$$

Note that given the parameters of the system, the right hand side of (7) can be regarded as an operation on the function $h$ which will be provided later in this section. This motivates us to define a fixed point function as follows.

Definition 2. We say $h$ is a fixed point function if for any choice of $\left(x_{i},\left\{y_{j i}\right\}_{j \in[n] \backslash\{i\}}\right)$ with $h\left(x_{i}\right)=z_{i}$, (7) also holds.

The following theorem is the main result of this section and presents a sufficient condition on $h$ to be a threshold function.

Theorem 3. Let $h: \mathbb{R} \rightarrow \mathbb{R}$ be strictly decreasing over $\mathbb{R}$ and also a fixed point function according to Definition 2. Then $h$ is a threshold function.

Proof. Let $y_{i}=\left(x_{i},\left\{y_{j i}\right\}_{j \in[n] \backslash\{i\}}\right)$ denote the observations of agent $i$. We need to consider two different cases: 
Case 1: $z_{i} \leqslant h\left(x_{i}\right)$. For any $k \in[n] \backslash\{i\}$, using Lemma 2, we have

$$
\begin{aligned}
& P\left(z_{k} \leqslant h\left(x_{k}\right) \mid y_{i}\right) \\
& =P\left(x_{i}+\varepsilon_{i k} \leqslant h\left(x_{k}\right)-\sum_{l \in[n] \backslash\{i, k\}} y_{l k} \mid y_{i}\right), \\
& =P\left(\varepsilon_{i k} \leqslant h\left(\overline{x_{k}}+\varepsilon_{k}\right)-x_{i}-\sum_{l \in[n] \backslash\{i, k\}}\left(\overline{y_{l k}}+\varepsilon_{l k}\right)\right),
\end{aligned}
$$

where $\overline{x_{k}}$ and $\overline{y_{l k}}$ are the mean of $x_{k}$ and $y_{l k}$ conditioned on $y_{i}$, respectively, and are derived in (3). Let $\epsilon=\left(\varepsilon_{k},\left\{\varepsilon_{l k}\right\}_{l \in[n] \backslash\{i, k\}}\right)$ and $f_{\epsilon}(\epsilon)$ denote the joint probability density function (PDF) of the Gaussian random variables $\left(\varepsilon_{k},\left\{\varepsilon_{l k}\right\}_{l \in[n] \backslash\{i, k\}}\right)$. By Lemma $2, \varepsilon_{i k}$ is independent from $\epsilon$ and thus, (8) can be rewritten as

$$
\begin{aligned}
& P\left(z_{k} \leqslant h\left(x_{k}\right) \mid y_{i}\right) \\
& =\int_{\mathbb{R}^{n-1}} \varphi\left(\frac{1}{\tau}\left(h\left(\overline{x_{k}}+\varepsilon_{k}\right)-x_{i}-\sum_{l \in[n] \backslash\{i, k\}}\left(\overline{y_{l k}}+\varepsilon_{l k}\right)\right)\right) f_{\epsilon}(\epsilon) d \epsilon
\end{aligned}
$$

where $\varphi$ is the cumulative distribution function (CDF) of the normal distribution with unit variance and $\tau$ is the variance of $\varepsilon_{i k}$, i.e., the noise variance of information exchange illustrated in Figure, 2. For $j \in[n] \backslash\{i\}$, let $y_{j i}^{\prime} \geqslant y_{j i}$ in such a way that $\sum_{j \in[n] \backslash\{i\}} y_{j i}^{\prime}=h\left(x_{i}\right)$. Let also ${\overline{x_{k}}}^{\prime}$ and $\bar{y}_{l k}$ be defined with respect to $x_{i}$ and $y_{j i}^{\prime}$ as in Lemma 2. Then by following the same arguments and by noting that $y_{i k}=x_{i}+\varepsilon_{i k}$ is kept the same by changing $y_{j i}$ to $y_{j i}^{\prime}$, we have

$$
\begin{aligned}
& P\left(z_{k} \leqslant h\left(x_{k}\right) \mid y_{i}^{\prime}\right) \\
& =\int_{\mathbb{R}^{n-1}} \varphi\left(\frac{1}{\tau}\left(h\left({\overline{x_{k}}}^{\prime}+\varepsilon_{k}\right)-x_{i}-\sum_{l \in[n] \backslash\{i, k\}}\left({\overline{y_{l k}}}^{\prime}+\varepsilon_{l k}\right)\right)\right) f_{\epsilon}(\epsilon) d \epsilon,
\end{aligned}
$$

where $y_{i}^{\prime}=\left(x_{i},\left\{y_{j i}^{\prime}\right\}_{j \in[n] \backslash\{i\}}\right)$. Observe that $\overline{x_{k}} \leqslant{\overline{x_{k}}}^{\prime}$ and $\overline{y_{l k}} \leqslant \bar{y}_{l k}^{\prime}$. Since $h$ is a decreasing function and $\varphi$ is an increasing function, (9) together with (10) imply that

$$
P\left(z_{k} \leqslant h\left(x_{k}\right) \mid y_{i}\right) \geqslant P\left(z_{k} \leqslant h\left(x_{k}\right) \mid y_{i}^{\prime}\right),
$$

By summing (11) over all $k \in[n] \backslash\{i\}$ and using the fixed point property of $h$ at $\left(x_{i},\left\{y_{j i}^{\prime}\right\}_{j \in[n] \backslash\{i\}}\right)$ as described in Definition 2, we get

$$
\begin{aligned}
& 1+\sum_{k \in[n] \backslash\{i\}} P\left(z_{k} \leqslant h\left(x_{k}\right) \mid y_{i}\right) \\
& \geqslant 1+\sum_{k \in[n] \backslash\{i\}} P\left(y_{k} \leqslant h\left(x_{k}\right) \mid y_{i}^{\prime}\right) \\
& \quad=a_{n} x_{i}+b_{n} h\left(x_{i}\right) \geqslant a_{n} x_{i}+b_{n} z_{i} \\
& =E\left[\theta \mid y_{i}\right] .
\end{aligned}
$$

Therefore, a best response of agent $i$ is to take the risky action.

Case 2: $z_{i}>h\left(x_{i}\right)$. Then we take $y_{j i}^{\prime}<y_{j i}$ in such a way that $\sum_{j \in[n] \backslash\{i\}} y_{j i}^{\prime}=h\left(x_{i}\right)$. The inequalities in (11) and (12) will be reversed and the best response of agent $i$ is to take the safe action. This will complete the proof.

\section{B. Convergence to the threshold function}

In this section, we analyze the convergence of an iterative scheme for finding the threshold function and consequently the threshold policy. For the sake of simplicity, through the rest of this section we limit our attention to the case of two agents. This simplifies the analysis of the fixed point function and the conditions we need to impose on the parameters of the system in order to guarantee the existence of the threshold functions. The results can be then generalized to the case of $n$ agents. Let

$$
\begin{array}{ll}
a=a_{2}=\frac{\sigma^{2}+\tau^{2}}{2 \sigma^{2}+\tau^{2}}, & b=b_{2}=\frac{\sigma^{2}}{2 \sigma^{2}+\tau^{2}}, \\
c=c_{2}=\frac{\tau^{2}}{2 \sigma^{2}+\tau^{2}}, & d=d_{2}=\frac{2 \sigma^{2}}{2 \sigma^{2}+\tau^{2}} .
\end{array}
$$

Note that the parameters $a, b, c, d$ are all linearly dependent on each other. For instance, the parameter $a$ is sufficient to know the other three parameters: $b=1-a, c=2 a-1$ and $d=2-2 a$. However, for clarity of discussion, we prefer to keep all the four parameters in the derivations.

For $n=2$, observe that for $x_{1}=x$ and $y_{1}=h(x)$, by Lemma 2 we have $\overline{x_{2}}=c x+d h(x)+\varepsilon$, where $\varepsilon \sim \mathcal{N}\left(0, \gamma_{2}^{2}\right)$. Therefore, using (9) in the proof of Theorem 3 , the definition of the fixed point function for the case of $n=2$ is re-stated as follows.

Definition 3. We call $h: \mathbb{R} \rightarrow \mathbb{R}$ to be a fixed point function if

$a x+b h(x)=1+\int_{-\infty}^{\infty} \varphi\left(\frac{h(c x+d h(x)+\varepsilon)-x}{\tau}\right) f_{\varepsilon}(\varepsilon) d \varepsilon$,

where $\varepsilon \sim \mathcal{N}\left(0, \gamma_{2}^{2}\right)$.

In order to find a threshold function, we are going to look for a fixed point function which is also decreasing over $\mathbb{R}$ which by Theorem 3 will be a threshold function.

In order to simplify the fixed point equation (13), let $g(x)=$ $a x+b h(x)-1$. Then $h(x)$ is a fixed point function if the following holds

$$
g(x)=\int_{-\infty}^{\infty} \varphi\left(\mathcal{M}_{\varepsilon} g(x)\right) f_{\varepsilon}(\varepsilon) d \varepsilon,
$$

where $\mathcal{M}_{\varepsilon}$ is an $\varepsilon$ dependent operator on $g: \mathbb{R} \rightarrow \mathbb{R}$ and $\mathcal{M}_{\varepsilon} g: \mathbb{R} \rightarrow \mathbb{R}$ is defined as

$$
\begin{aligned}
\mathcal{M}_{\varepsilon} g(x) & :=\frac{1}{b \tau} g(2 g(x)-x+\varepsilon+2) \\
& +\frac{1}{b \tau}(-2 a g(x)+(2 a-1) x-a \varepsilon-2 a+1) .
\end{aligned}
$$

Definition 4. We define the operator $\mathcal{T}$ to be the operator that maps a sufficiently well-behaved function $g: \mathbb{R} \rightarrow \mathbb{R}$ to $\mathcal{T} g:$ $\mathbb{R} \rightarrow \mathbb{R}$ defined by

$$
\mathcal{T} g(x):=\int_{-\infty}^{\infty} \varphi\left(\mathcal{M}_{\varepsilon} g(x)\right) f_{\varepsilon}(\varepsilon) d \varepsilon
$$

where $\mathcal{M}_{\varepsilon} g$ is defined in (15).

In the subsequent discussion, we will derive conditions that will characterize the term sufficiently well-behaved in the above statement. First we notice that $\mathcal{T}$ can be viewed as a mapping that maps the space of measurable functions on $\mathbb{R} \rightarrow[0,1]$ to itself. This follows from the fact that $\varphi(\alpha) \in$ $[0,1]$ for all $\alpha \in \mathbb{R}$. 
Observe that if $\mathcal{T} g \equiv g$, then $h(x)=\frac{g(x)}{b}-\frac{a x}{b}+\frac{1}{b}$ is a fixed point function. Therefore, finding a fixed point function $h$ is equivalent to finding a fixed point for the operator $\mathcal{T}$.

To find such a fixed point, the structure of the fixed point equation (16) suggests the investigation of the iterations

$$
g^{(t+1)}=\mathcal{T} g^{(t)},
$$

for some sufficiently well-behaved initial function $g^{(0)}$. Indeed, we will prove that $\mathcal{T}$ induces a contraction mapping on the space $C_{0}(\mathbb{R},[0,1])$ of continuous functions from $\mathbb{R}$ to $[0,1]$ and hence, converges to a unique fixed point.

In order to make sure that $g$ leads to a threshold function $h$, we need to show that the property of being strictly decreasing on $h$ can be preserved through the iteration. Therefore, the stronger condition of Lipschitz continuity is imposed on $g$, i.e., we require that for any $x_{1}, x_{2} \in \mathbb{R}$ :

$$
\left|g\left(x_{1}\right)-g\left(x_{2}\right)\right| \leqslant a\left|x_{1}-x_{2}\right|,
$$

Let $\mathcal{F}$ denote the space of all Lipschitz continuous functions $f$ with parameter $a$. We aim at characterizing conditions under which the Lipschitz continuity is preserved through the operation $\mathcal{T}$. In other words $\mathcal{T}(\mathcal{F}) \subseteq \mathcal{F}$, where $\mathcal{T}(\mathcal{F})=$ $\{\mathcal{T}(h): h \in \mathcal{F}\}$. This is done in the next lemma.

Lemma 4. If

$$
\frac{4 a^{2}+3 a-1}{a(1-a)} \leqslant \tau,
$$

then Lipschitz continuity with parameter $a$ is preserved through the operation $\mathcal{T}$.

Proof. Let $x_{1}, x_{2} \in \mathbb{R}$. Then by using the definition of operation $\mathcal{T}$ we have

$$
\begin{aligned}
& \left|\mathcal{T} g\left(x_{1}\right)-\mathcal{T} g\left(x_{2}\right)\right| \\
& \leqslant \int_{-\infty}^{\infty}\left|\varphi\left(\mathcal{M}_{\varepsilon} g\left(x_{1}\right)\right)-\varphi\left(\mathcal{M}_{\varepsilon} g\left(x_{2}\right)\right)\right| f_{\varepsilon}(\varepsilon) d \varepsilon \\
& \leqslant \int_{-\infty}^{\infty}\left|\mathcal{M}_{\varepsilon} g\left(x_{1}\right)-\mathcal{M}_{\varepsilon} g\left(x_{2}\right)\right| f_{\varepsilon}(\varepsilon) d \varepsilon,
\end{aligned}
$$

where we used the fact that $|\varphi(A)-\varphi(B)| \leqslant|A-B|$, for any $A, B \in \mathbb{R}$, in the last inequality. Therefore, Let $\delta=$ $\left|x_{1}-x_{2}\right|$. In the following series of inequalities, we only use the Lipschitz continuity of $g$ as given in (18) and triangle inequality.

$$
\begin{aligned}
& b \tau\left|\mathcal{M}_{\varepsilon} g\left(x_{1}\right)-\mathcal{M}_{\varepsilon} g\left(x_{2}\right)\right| \\
& \leqslant\left|g\left(2 g\left(x_{1}\right)-x_{1}+\varepsilon\right)-g\left(2 g\left(x_{2}\right)-x_{2}+\varepsilon\right)\right| \\
& +2 a\left|g\left(x_{1}\right)-g\left(x_{2}\right)\right|+(2 a-1) \delta \\
& \leqslant a\left|2 g\left(x_{1}\right)-x_{1}-2 g\left(x_{2}\right)+x_{2}\right|+2 a^{2} \delta+(2 a-1) \delta \\
& \leqslant 2 a\left|g\left(x_{1}\right)-g\left(x_{2}\right)\right|+a\left|x_{1}-x_{2}\right|+2 a^{2} \delta+(2 a-1) \delta \\
& \leqslant 4 a^{2} \delta+(3 a-1) \delta .
\end{aligned}
$$

This together with (20) imply that

$$
\left|\mathcal{T} g\left(x_{1}\right)-\mathcal{T} g\left(x_{2}\right)\right| \leqslant \frac{4 a^{2}+3 a-1}{b \tau}\left|x_{1}-x_{2}\right|
$$

Therefore, a sufficient condition for $\mathcal{T} g$ for being Lipschitz continuous with parameter $a$ is that

$$
\frac{4 a^{2}+3 a-1}{b \tau} \leqslant a .
$$

Note that $b=1-a$ which completes the proof.
Suppose that the parameters of the system satisfy (19). First notice that since $\varphi(\alpha) \in[0,1]$, it follow that $\mathcal{T} g(x) \in$ $[0,1]$ for all $x \in \mathbb{R}$. Therefore, one can view $\mathcal{T}$ as a mapping $\mathcal{T}: \mathcal{F} \cap C_{0}(\mathbb{R},[0,1]) \rightarrow \mathcal{F} \cap C_{0}(\mathbb{R},[0,1])$ where $C_{0}(\mathbb{R},[0,1])$ is the space of continuous functions from $\mathbb{R}$ to $[0,1]$ embedded with the uniform norm ||$g-h \|:=\sup _{x \in \mathbb{R}}|g(x)-h(x)|$. The following lemma shows that indeed such a mapping is a contraction mapping for a set of parameters.

Lemma 5. For any two functions $g, h \in \mathcal{T}(\mathcal{F})$,

$$
\|\mathcal{T} g-\mathcal{T} h\| \leqslant \frac{4 a+1}{(1-a) \tau}\|g-h\| .
$$

Proof. For any $x \in \mathbb{R}$,

$$
\begin{aligned}
& \mathcal{T} g(x)-\mathcal{T} h(x)=\int_{-\infty}^{\infty}\left(\varphi\left(\mathcal{M}_{\varepsilon} g(x)\right)-\varphi\left(\mathcal{M}_{\varepsilon} h(x)\right)\right) f_{\varepsilon}(\varepsilon) d \varepsilon \\
& \leqslant \int_{-\infty}^{\infty} \mid \mathcal{M}_{\varepsilon} g(x)-\left(\mathcal{M}_{\varepsilon} h(x) \mid f_{\varepsilon} d \varepsilon\right. \\
& \leqslant \sup _{\varepsilon \in \mathbb{R}}\left(\mid \mathcal{M}_{\varepsilon} g(x)-\left(\mathcal{M}_{\varepsilon} h(x) \mid\right) .\right.
\end{aligned}
$$

For any $\varepsilon \in \mathbb{R}$, by definition of $\mathcal{M}_{\varepsilon} g$ and $\mathcal{M}_{\varepsilon} h$ in (15) and triangle inequality we have

$$
\begin{aligned}
& b \tau\left(\mathcal{M}_{\varepsilon} g(x)-\left(\mathcal{M}_{\varepsilon} h(x)\right)\right. \\
= & |g(2 g(x)-x+\varepsilon+2)-h(2 h(x)-x+\varepsilon+2)| \\
& +2 a|g(x)-h(x)| \\
\leqslant & |g(2 g(x)-x+\varepsilon+2)-g(2 h(x)-x+\varepsilon+2)| \\
& +|g(2 h(x)-x+\varepsilon+2)-h(2 h(x)-x+\varepsilon+2)| \\
& +2 a|| g-h \| \\
\leqslant & 2 a|g(x)-h(x)|+(2 a+1)|| g-h\|\leqslant(4 a+1)\| g-h \|,
\end{aligned}
$$

where we only used Lemma 4 for Lipschitz continuity of $g$ and $h$ with parameter $a$. The proof is complete by noting that $b=1-a$.

Theorem 6. Let $\frac{4 a+1}{1-a}<\tau$, then the sequence of functions $\left\{g^{(t)}\right\}$ defined by (17) converges to a unique fixed point $g=$ $\mathcal{T} g$ in the space $\mathcal{L}_{\infty}(\mathbb{R})$, where $\mathcal{L}_{\infty}(\mathbb{R})$ is the set space of (Lebesgue) measurable functions over $\mathbb{R}$ with the $\mathcal{L}_{\infty}$ norm.

Proof. Let $M=\frac{4 a+1}{b \tau}<1$. Then by Lemma 5, $\mathcal{T}$ would be a contraction mapping over $\mathcal{L}_{\infty}(\mathbb{R})$ (which is a complete space) and hence, the result follows immediately by the Banach Fixed Point Theorem [10].

In order to satisfy the requirements for a threshold function, we need both the conditions derived in Lemma 4 and Theorem 6 to be satisfied. Since $2 a \geqslant 1$, we have

$$
\frac{4 a^{2}+3 a-1}{a(1-a)} \geqslant \frac{4 a^{2}+a}{a(1-a)}=\frac{4 a+1}{1-a} .
$$

Therefore, we get the following theorem.

Theorem 7. If

$$
\frac{4 a^{2}+3 a-1}{a(1-a)} \leqslant \tau,
$$

then there exists a continuous threshold function $h: \mathbb{R} \rightarrow \mathbb{R}$. Furthermore, $h(x)$ can be numerically approximated with arbitrarily enough precision, with respect to $\mathcal{L}_{\infty}$ norm.

Proof. The proof follows from Theorem 3, Lemma 4 and Theorem 6. The continuity of $g$ (and hence, $h$ ) follows from the fact that if the above condition holds, then $\mathcal{T}$ would be a 

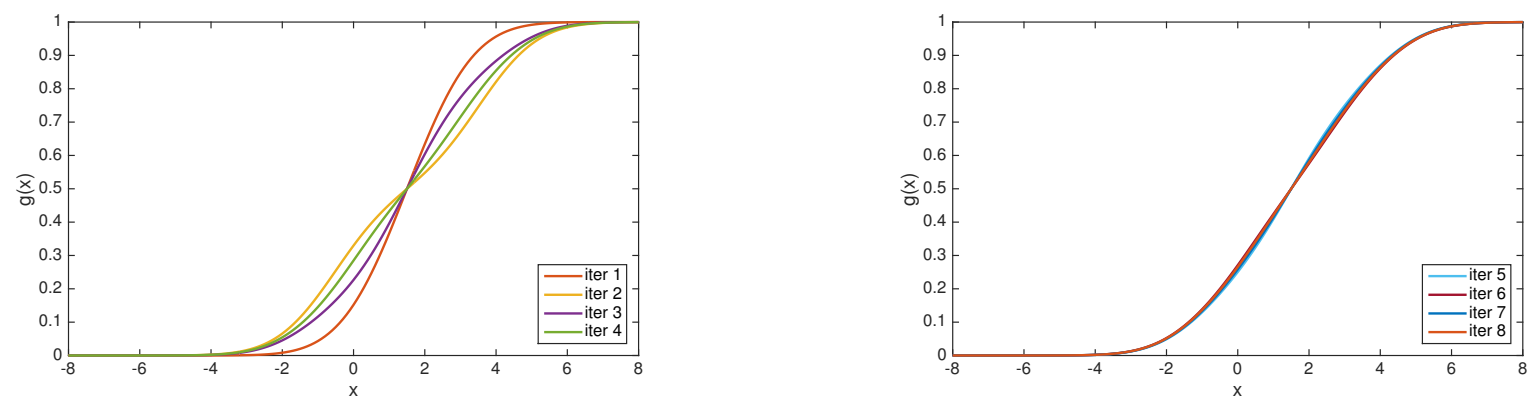

Figure 3. The iterative solution $g(x)$ of equation (14) for $\sigma^{2}=1$ and $\tau^{2}=9$.

contraction mapping from $\mathcal{F} \cap C_{0}(\mathbb{R},[0,1])$ to itself and $\mathcal{F} \cap$ $C_{0}(\mathbb{R},[0,1])$ is a closed subset $C_{0}(\mathbb{R},[0,1])$. Therefore, the iterations converge to a function in $\mathcal{F}$ which is (Lipschitz) continuous.

\section{Discussions and simulation results}

In this section, we proved that there is a unique coordinated equilibrium strategy of the form $\left(1_{y_{1} \leqslant h\left(x_{1}\right)}, 1_{y_{2} \leqslant h\left(x_{2}\right)}\right)$ for some continuous and strictly decreasing function $h$, under the condition provided in Theorem 7 . Note that for a fixed ratio of $r=\frac{\tau}{\sigma}, a=\frac{r^{2}+1}{r^{2}+2}$ is also fixed and hence, for $\tau$ greater than the lower bound of Theorem 7, which can be described in terms of $r$, the threshold policy exists.

One can derive similar conditions for threshold policies of the form $\left(1_{x_{1} \leqslant f\left(y_{1}\right)}, 1_{x_{2} \leqslant f\left(y_{2}\right)}\right)$. Since $h$ is a decreasing continuous function, $h^{-1}$ exists and hence, $f=h^{-1}$ becomes a solution for the later problem, i.e., the policy can be also described by $1_{x_{i} \leqslant h^{-1}\left(y_{i}\right)}$. However, the resulting conditions on the noise variances of the system for the later problem will be different. This is not a contradiction with our earlier findings as we believe that these conditions are not tight and hence, an interesting question would be to get the best sufficient condition that guarantees the existence of such a threshold policy equilibrium.

In Figure 3, we also provide simulation results and examine the coordinated threshold policy for the case of $n=2$. In the simulations, we demonstrate the convergence of solution $g(x)$ of (14) after only eight iterations. Recall that the threshold function is

$$
h(x)=\frac{1}{b} g(x)-\frac{a}{b} x+\frac{1}{b},
$$

which is close to linear for large values of $|x|$.

\section{CONClusion And Future Work}

In this paper, we studied global games with noisy sharing of information and the existence and the structure of equilibria for those games. We showed that under some conditions on the noise parameters of the system, there exists a unique threshold-type equilibrium policy. To show this, we formulated the existence of such an equilibrium as a existence of a solution to a functional fixed point equation. Using this, we showed that for a set of noise parameters, the fixed point equation results in a contraction mapping on certain complete spaces and hence, we obtain a unique equilibrium based on threshold policies. Furthermore, such a contraction mapping would also provide us with a natural approximation mechanism.
Many questions are left open including the study of global games with noisy sharing of information under more general structure. Also, it is not clear whether or not there exists an equilibrium policy for noise parameters $\sigma^{2}$ and $\tau^{2}$ that do not satisfy the stated conditions for our results.

\section{APPENDIX}

Lemma 8. Let $\theta$ be picked from the non-informative uniform prior on $\mathbb{R}$. Further, let $\left\{\phi_{i}\right\}_{i \in[n]}$ denote noisy observations of $\theta$ with additive zero-mean independent Gaussian noises with variances $\left\{\sigma_{i}^{2}\right\}_{i \in[n]}$. Let $\frac{1}{\sigma_{\theta}^{2}}:=\sum_{i \in[n]} \frac{1}{\sigma_{i}^{2}}$. Then, conditioned on $\left\{\phi_{i}\right\}_{i \in[n]}$, we can write $\theta$ as follows.

$$
\theta=\sigma_{\theta}^{2} \sum_{i \in[n]} \frac{\phi_{i}}{\sigma_{i}^{2}}+z
$$

where $z$ is $\mathcal{N}\left(0, \sigma_{\theta}^{2}\right)$ which is independent of $\left\{\phi_{i}\right\}_{i \in[n]}$.

Proof. We have

$$
\begin{aligned}
f\left(\theta \mid\left\{\phi_{i}\right\}_{i \in[n]}\right) & =\frac{f\left(\left\{\phi_{i}\right\}_{i \in[n]} \mid \theta\right)}{\int_{-\infty}^{\infty} f\left(\left\{\phi_{i}\right\}_{i \in[n]} \mid \alpha\right) d \alpha} \\
& =\frac{\exp \left(-\sum_{i \in[n]} \frac{\left(\phi_{i}-\theta\right)^{2}}{2 \sigma_{i}^{2}}\right)}{\int_{-\infty}^{\infty} \exp \left(-\sum_{i \in[n]} \frac{\left(\phi_{i}-\alpha\right)^{2}}{2 \sigma_{i}^{2}}\right) d \alpha} .
\end{aligned}
$$

By inspecting the PDF in (22), it is clear that $\theta$ is a Gaussian random variable conditioned on $\left\{\phi_{i}\right\}_{i \in[n]}$. It is therefore sufficient to calculate its mean and its variance, which lead to the desired result.

\section{REFERENCES}

[1] B. Touri and J. Shamma, "Global games with noisy sharing of information," in 53rd IEEE CDC, 2014, pp. 4473-4478.

[2] H. Carlsson and E. van Damme, "Global games and equilibrium selection," Econometrica, vol. 61, pp. 989-1018, 1993.

[3] S. Morris and H. S. Shin, "Unique equilibrium in a model of selffulfilling currency attacks," American Economic Review, vol. 88, p. $587=597,1998$.

[4] — , "Global games: theory and applications," Econometric Society Monographs, vol. 35, pp. 56-114, 2003.

[5] M. Dahleh, A. Tahbaz-Salehi, J. Tsitsiklis, and S. Zoumpoulis, "On global games in social networks of information exchange," 2012.

[6] A. Nayyar, A. Mahajan, and D. Teneketzis, "Optimal control strategies in delayed sharing information structures," Automatic Control, IEEE Transactions on, vol. 56, no. 7, pp. 1606-1620, 2011.

[7] A. Gupta, S. Yuksel, T. Basar, and C. Langbort, "On the existence of optimal policies for a class of static and sequential dynamic teams," arXiv preprint arXiv:1404.1404, 2014.

[8] A. Mahajan, N. Martins, M. Rotkowitz, and S. Yuksel, "Information structures in optimal decentralized control," in 51st IEEE CDC, Dec 2012, pp. 1291-1306.

[9] J. Marden, "The role of information in multiagent coordination," in 53rd IEEE CDC, Dec 2014, pp. 445-450.

[10] H. K. Khalil and J. Grizzle, Nonlinear systems. Prentice hall Upper Saddle River, 2002, vol. 3. 